\title{
Primeiro encontro de Aedes (Stegomyia) albopictus (Skuse) no Estado do Paraná, Brasil
}

\section{First recorded occurrence of Aedes (Stegomyia) albopictus (Skuse) in the South-Eastern region of Brazil}

\author{
Adson Luís Sant'Ana \\ Departamento de Zoologia da Universidade Federal do Paraná. Curitiba, PR - Brasil
}

\begin{abstract}
Resumo
Foi constatada a primeira ocorrência de Aedes (Stegomyia) albopictus (Skuse) na cidade de Curitiba, Paraná. A coleta foi realizada, utilizando um sugador e isca humana como atração.

Aedes.

Abstract

The first recorded occurrence of Aedes (Stegomyia) albopictus (Skuse) in Curitiba, Paraná, South of Brazil, was described. The collection was carried out by means of aspirator in human bait.
\end{abstract}

Aedes.

Foram encontrados dois adultos fêmeas de Aedes albopictus, um no dia 17 de abril de 1996 e outro no dia 3 de maio de 1996, na cidade de Curitiba, Paraná. As duas fêmeas foram coletadas com sugador, utilizando isca humana em uma residência próxima à BR277, ao lado do Centro Politécnico da Universidade Federal do Paraná, tendo a identificação sido confirmada pelo laboratório de Entomologia da Faculdade de Saúde Pública da Universidade de São Paulo.

A cidade de Curitiba está situada ao centro-sul do primeiro planalto paranaense. De acordo com Bigarella e Salamuni ${ }^{1,2}(1957,1959)$, o Planalto de Curitiba está situado na região oriental do Estado, estendendo-se de norte a sul, em forma de arco, com uma largura de 70 a $80 \mathrm{~km}$, limitando-se a leste pela Serra do Mar e a oeste pela Escarpa Devoniana. A bacia de Curitiba ocupa uma área de cerca de 3.000 $\mathrm{km}^{2}$, na parte meridional do Planalto. Na parte central deste as cotas altimétricas são de 930 a $935 \mathrm{~m}$.

O Aedes albopictus é um potencial vetor da dengue, apresentando uma ampla distribuição no sul da ásia Oriental e no Pacífico Sul. A primeira identificação de Aedes albopictus, no Brasil, foi feita por Forattini $^{4}$ (1986) a partir de exemplares encontrados no Estado do Rio de Janeiro. Acredita-se que a origem desses focos tenha sido o Japão, através do intercâmbio marítimo desse país com o sistema portuário do Estado do Espírito Santo (Companhia Vale do Rio Doce). Sua ocorrência no Estado de São Paulo, no Município de Areias (Vale do Paraíba), foi relatada por Brito e col. ${ }^{3}$, (1986). Também foi referido no Município de Tremembé por Gomes e Marques ${ }^{5}$ (1988) e na cidade de São Paulo por Soubihe e col. ${ }^{6}$, (1992). 


\section{AGRADECIMENTO}

À Professora Dra . Maria Anice Mureb Sallum, do Departamento de Epidemiologia da Faculdade de

\section{REFERÊNCIAS BIBLIOGRÁFICAS}

1. BIGARELLA, J.J. \& SALAMUNI, R. Aspectos geográficos e geológicos da cidade de Curitiba. Cad. Miner. e Petr. Geol. Fac. Cat. Filos., 1957.

2. BIGARELLA, J. J. \& SALAMUNI, R. Notas complementares à planta geológica da cidade de Curitiba $\mathrm{e}$ arredores. Inst. Biol. Pesq. Tecnol., 40: 1-14, 1959.

3. BRITO, A. et al. Primeiro encontro de Aedes (Stegomyia) albopictus (Skuse) no Estado de São Paulo, Brasil. Rev. Saúde Pública, 20: 489, 1986.
Saúde Pública (Núcleo de Pesquisa Taxonômica e Sistemática em Entomologia Médica) da Universidade de São Paulo, pela confirmação na identificação do exemplar de Aedes albopictus.

4. FORATTINI, O.P. Identificação de Aedes (Stegomyia) albopictus (Skuse) no Brasil. Rev. Saúde Pública, 20: 244-5, 1986.

5. GOMES, A.C. \& MARQUES, G.R.A.M. Encontro de criadouro natural de Aedes (Stegomyia) albopictus (Skuse), no Estado de São Paulo, Brasil. Rev. Saúde Pública, 22: 245, 1988.

6. SOUBIHE, V. et al. Presença de Aedes (Stegomyia) albopictus (Skuse) na cidade de São Paulo-SP, Brasil. Rev. Saúde Pública, 26: 57, 1992. 